\title{
Analyte Type
}

National Cancer Institute

\section{Source}

National Cancer Institute. Analyte Type. NCI Thesaurus. Code C156434.

The molecular type that describes the biospecimen used in a study. 